\title{
On the formal structure of logarithmic vector fields
}

\author{
Michel Granger and Mathias Schulze
}

\begin{abstract}
In this article, we prove that a free divisor in a three-dimensional complex manifold must be Euler homogeneous in a strong sense if the cohomology of its complement is the hypercohomology of its logarithmic differential forms. Calderón-Moreno et al. conjectured this implication in all dimensions and proved it in dimension two. We prove a theorem that describes in all dimensions a special minimal system of generators for the module of formal logarithmic vector fields. This formal structure theorem is closely related to the formal decomposition of a vector field by Kyoji Saito and is used in the proof of the above result. Another consequence of the formal structure theorem is that the truncated Lie algebras of logarithmic vector fields up to dimension three are solvable. We give an example that this may fail in higher dimensions.
\end{abstract}

\section{Introduction}

Let $X$ be a complex manifold of dimension $n \geqslant 1$. Let $D \subseteq X$ be a reduced divisor and $j: U \hookrightarrow X$ the inclusion of its complement $U:=X \backslash D$ in $X$. By Grothendieck's comparison theorem [Gro66, Theorem 2], the natural morphism

$$
\Omega_{X}^{\bullet}(* D) \longrightarrow j_{*} \Omega_{U}^{\bullet} \simeq \mathbf{R} j_{*} \mathbb{C}_{U}
$$

is a quasi-isomorphism. This means that the complex $\Omega_{X}^{\bullet}(* D)$ of holomorphic differential forms with meromorphic poles along $D$ can be used to calculate the cohomology of $U$.

Generalizing ideas of Deligne and Katz, Saito [Sai80] defined the subcomplex $\Omega_{X}^{\bullet}(\log D) \subseteq$ $\Omega_{X}^{\bullet}(* D)$ of holomorphic differential forms with logarithmic poles along $D$. Unlike $\Omega_{X}^{\bullet}(* D)$, $\Omega_{X}^{\bullet}(\log D)$ is a complex of coherent $\mathcal{O}_{X}$-modules. If $D$ is a normal crossing divisor, $\Omega_{X}^{\bullet}(\log D)$ also computes the cohomology of $U$. This fact plays a crucial role in Deligne's mixed Hodge theory [Del71, §3]. In general, one says that the logarithmic comparison theorem (LCT), holds for $D$ if the inclusion

$$
\Omega_{X}^{\bullet}(\log D) \longleftrightarrow \Omega_{X}^{\bullet}(* D)
$$

is a quasi-isomorphism. The characterization of LCT in general is an open problem.

The natural dual of $\Omega_{X}^{1}(\log D)$ is the module $\operatorname{Der}_{X}(-\log D)$ of $\log$ arithmic vector fields along $D$. As recently proposed by Saito, we adopt a notation harmonized with the conventions of algebraic geometry. For $x \in X$, a vector field $\delta \in \operatorname{Der}_{X, x}$ is contained in $\operatorname{Der}_{X, x}(-\log D)$ if $\delta(f) \in \mathcal{O}_{X, x} \cdot f$ for some, and hence any, (reduced) local equation $f \in \mathcal{O}_{X, x}$ of $(D, x)$. We often use the standard notation $\mathrm{I}(D, x)=\langle f\rangle:=\mathcal{O}_{X, x} \cdot f$ or $(D, x)=\mathrm{V}(f)$. Saito [Sai80] introduced the important class of free divisors: the divisor $D$ is called free if $\Omega_{X}^{1}(\log D)$, or equivalently $\operatorname{Der}_{X}(-\log D)$, is a locally

Received 1 February 2005, accepted in final form 16 September 2005.

2000 Mathematics Subject Classification 32S65, 32S20, 14F40, $17 \mathrm{~B} 66$.

Keywords: free divisor, logarithmic vector field, Euler homogeneity, de Rham cohomology, logarithmic comparison theorem.

The second author was supported by ÉGIDE.

This journal is (C) Foundation Compositio Mathematica 2006. 


\section{Granger And M. Schulze}

free $\mathcal{O}_{X}$-module. Prominent examples of free divisors are normal crossing divisors or discriminants of stable mappings $f: X \longrightarrow Y$ where $\operatorname{dim} X \geqslant \operatorname{dim} Y$.

A holomorphic function $f \in \mathcal{O}_{X, x}$ is called Euler homogeneous if $\chi(f)=f$ for some $\chi \in \operatorname{Der}_{X, x}$ which is then called an Euler vector field for $f$. We call $f$ strongly Euler homogeneous at $x$ if it admits an Euler vector field $\chi \in \mathfrak{m}_{X, x} \cdot \operatorname{Der}_{X, x}$. The divisor $D$ is called Euler homogeneous if, for all $x \in D, \mathrm{I}(D, x)=\langle f\rangle$ for some Euler homogeneous $f \in \mathcal{O}_{X, x}$. We call $D$ strongly Euler homogeneous at $x$ if $\mathrm{I}(D, x)=\langle f\rangle$ for some strongly Euler homogeneous $f \in \mathcal{O}_{X, x}$ at $x$. By Lemma 3.2, a product $(D, x)=\left(D^{\prime}, x^{\prime}\right) \times(\mathbb{C}, 0)$ with a smooth factor is Euler homogeneous and strong Euler homogeneity of $(D, x)$ and $\left(D^{\prime}, x^{\prime}\right)$ are equivalent. By strong Euler homogeneity of $D$ we mean strong Euler homogeneity at $x$ for all $x \in D$. This definition can be fulfilled by a single local Euler vector field only at smooth points. Whereas Euler homogeneity is obviously an open condition, strong Euler homogeneity is not. For example, the free divisor $D=\mathrm{V}\left(z\left(x^{4}+x y^{4}+y^{5}\right)\right) \subseteq \mathbb{C}^{3}$ (see $\left.[\mathrm{CU} 05, \S 1]\right)$ is strongly Euler homogeneous at 0, with Euler vector field $\chi=z \partial_{z}$, but not at the other points of the $z$-axis by Lemma 3.2 and Saito [Sai71].

The divisor $D$ is called locally quasi-homogeneous if, for all $x \in D,(D, x)$ is defined by a quasihomogeneous polynomial with respect to strictly positive weights in some local coordinate system centered at $x$. Local quasi-homogeneity obviously implies (strong) Euler homogeneity. By Saito [Sai71], the three properties are equivalent if $D$ has only isolated singularities or, in particular, in dimension $n=2$.

By Castro-Jiménez et al. [CNM96], local quasi-homogeneity implies LCT for free divisors. Calderón-Moreno et al. [CMNC02, Theorem 1.3] prove that equivalence holds in dimension $n=2$ by an explicit technical construction of an Euler vector field [CMNC02, Theorem 3.3]. In [CMNC02, Conjecture 1.4], they propose the following generalization of their result to higher dimensions, which is the main motivation for this article.

Conjecture 1.1 (Calderón-Moreno et al. [CMNC02]). Let $D$ be a free divisor in a complex manifold $X$. If the logarithmic comparison theorem holds for $D$, then $D$ is strongly Euler homogeneous.

Theorem 1.2 (Calderón-Moreno et al. [CMNC02]). Conjecture 1.1 holds in dimension $n=2$.

For any dimension $n \geqslant 3$, Castro-Jiménez and Ucha-Enríquez [CU05] found a family of Euler homogeneous free divisors for which LCT does not hold. However, these divisors are not strongly Euler homogeneous as any Euler vector field at a point in $D$ with $x_{1}=0=x_{2}$ and $x_{n} \neq 0$ has a non-vanishing $\partial_{n}$ component. Thus, the converse of Conjecture 1.1 is an open problem as well.

Saito [Sai80, §3] constructed the logarithmic stratification of a divisor by integration along logarithmic vector fields. At each point of a logarithmic stratum, the logarithmic vector fields span the tangent space of this stratum. In his language, a divisor may be called holonomic if this stratification is locally finite or, equivalently, its logarithmic characteristic variety is of minimal dimension $n$ (see [Sai80, Proposition 3.18]). A divisor $D$ is called Koszul free at $x$ if an $\mathcal{O}_{X, x^{-b a s i s}}$ of $\operatorname{Der}_{X, x}(-\log D)$ defines a regular sequence in $\operatorname{gr}^{F} \mathcal{D}_{X, x}$ where $F_{\bullet}$ denotes the filtration by order on the ring of differential operators $\mathcal{D}_{X}$ (see [CN02, Definition 1.6]). Koszul free is the same as free holonomic in the above sense [CN02, Corollary 1.9]. For example, the free divisor $D=\mathrm{V}(x y(x+y)$ $(x z+y)) \subseteq \mathbb{C}^{3}$ (see [CN02, Example 6.2]) fulfills LCT but is not Koszul free as any point of the $z$-axis is a logarithmic stratum.

Conjecture 1.1 is the missing piece in the following diagram of known implications and nonimplications for a free divisor $[\mathrm{CN} 02, \S 6]$.

locally quasi-homogeneous $\Longleftrightarrow$ Euler homogeneous

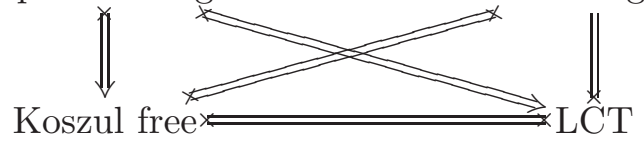




\section{ON THE FORMAL STRUCTURE OF LOGARITHMIC VECTOR FIELDS}

Calderón-Moreno and Narváez-Macarro [CN05, Corollaire 4.3] recently gave the following characterization of LCT for free divisors.

Theorem 1.3 (Calderón-Moreno and Narváez-Macarro [CN05]). Let $D$ be a free divisor in a complex manifold $X$. Then LCT holds for $D$ if and only if the logarithmic Spencer complex

$$
\mathcal{D}_{X} \stackrel{\mathrm{L}}{\otimes}_{\mathcal{D}_{X}(\log D)} \mathcal{O}_{X}(D) \simeq \mathcal{D}_{X} \otimes_{\mathcal{D}_{X}(\log D)} \operatorname{Sp}_{\mathcal{D}_{X}(\log D)}\left(\mathcal{O}_{X}(D)\right)
$$

is concentrated in degree 0 and the natural morphism

$$
\mathcal{D}_{X} \otimes_{\mathcal{D}_{X}(\log D)} \mathcal{O}_{X}(D) \stackrel{\epsilon_{D}}{\longrightarrow} \mathcal{O}_{X}(* D)
$$

is injective.

The injectivity of $\epsilon_{D}$ in Theorem 1.3 at $x \in D$ where $\mathrm{I}(D, x)=\langle f\rangle$ and $f \in \mathfrak{m}_{X, x}$ means exactly that the annihilator $\operatorname{Ann}_{\mathcal{D}_{X, x}}(1 / f)$ is generated by differential operators of order one. For Koszul free divisors, the first condition in Theorem 1.3 is fulfilled and the second was characterized by Torrelli [Tor04, Theorem 1.7] as follows.

Theorem 1.4 (Torrelli [Tor04]). Let $0 \neq f \in \mathfrak{m}_{X, x}$ be a Koszul free germ. Then $\mathrm{Ann}_{\mathcal{D}_{X, x}}(1 / f)$ is generated by differential operators of order one if and only if $f$ is Euler homogeneous, -1 is the smallest integer root of the Bernstein polynomial of $f$, and the annihilator $\operatorname{Ann}_{\mathcal{D}_{X, x}}\left(f^{s}\right)$ is generated by differential operators of order one.

In particular, LCT implies Euler homogeneity for Koszul free divisors. We only need this implication but one can easily deduce the following stronger statement.

Corollary 1.5. Conjecture 1.1 holds for Koszul free divisors.

Proof. We may identify $(X, x)=\left(\mathbb{C}^{n}, 0\right)$ and assume that LCT holds for the Koszul free divisor $(D, 0)=\mathrm{V}(f) \subseteq\left(\mathbb{C}^{n}, 0\right)$ where $f \in \mathcal{O}_{\mathbb{C}^{n}, 0}$. By Theorems 1.3 and 1.4, we know that $(D, 0)$ is already Euler homogeneous. If $(D, 0)$ is not strongly Euler homogeneous then we may assume that there is an Euler vector field $\chi \in \operatorname{Der}_{\mathbb{C}^{n}, 0} \backslash \mathfrak{m}_{\mathbb{C}^{n}, 0} \cdot \operatorname{Der}_{\mathbb{C}^{n}, 0}$ for $f$. By Saito [Sai80, Lemma 3.5], integration along $\chi$ yields a coordinate system $\underline{x}=\left(x_{1}, \ldots, x_{n}\right)$ such that $f=u \cdot f^{\prime}$ where $u \in \mathcal{O}_{\mathbb{C}^{n}, 0}^{*}$ and $f=f\left(x_{1}, \ldots, x_{n-1}\right) \in \mathcal{O}_{\mathbb{C}^{n-1}, 0}$. This means that $(D, 0)=(D, 0)^{\prime} \times(\mathbb{C}, 0)$ where $\left(D^{\prime}, 0\right)=\mathrm{V}^{\prime}\left(f^{\prime}\right) \subseteq$ $\left(\mathbb{C}^{n-1}, 0\right)$ and Koszul freeness, strong Euler homogeneity, and LCT for $D$ and $D^{\prime}$ are equivalent by Lemmas 7.3 and 7.4. However, the condition on the annihilator in Theorem 1.4 is also equivalent for $f$ and $f^{\prime}$. This is a contradiction by induction on the dimension $n$.

In this article, we describe the formal structure of the logarithmic vector fields, that is the $\mathfrak{m}_{X, x}$-adic completion of $\operatorname{Der}_{X, x}(-\log D)$. The result in Theorem 5.4 is obtained by performing the construction of Saito [Sai71, §3] of the Poincaré-Dulac decomposition [AA88, ch. 3, § 3.2] simultaneously to a system of generators. In Theorem 1.6, we combine this result with an explicit necessary condition for LCT for a free divisor due to Calderón-Moreno et al. [CMNC02, § 2] to prove our main result.

Theorem 1.6. Conjecture 1.1 holds in dimension $n=3$.

In fact, it turns out that this problem is purely formal for a non-Koszul free divisor.

As a further application of Theorem 5.4, we describe in Propositions 6.1 and 6.2 the formal Lie algebra structure of the logarithmic vector fields for a free divisor in dimension $n \leqslant 3$. In Example 6.3, we give a counter-example in dimension $n=4$. 


\section{Granger And M. Schulze}

\section{Vector fields}

We denote row vectors by a lower bar and column vectors by an upper bar. Let $\mathcal{O}:=\mathcal{O}_{n}:=\mathbb{C}\{\underline{x}\}$ be the ring of convergent power series in the variables $\underline{x}=\left(x_{1}, \ldots, x_{n}\right)$ and $\mathfrak{m}:=\mathfrak{m}_{n}:=\langle\underline{x}\rangle$ its maximal ideal. There are analog definitions and statements as in this section for the ring $\widehat{\mathcal{O}}=\mathbb{C} \llbracket \underline{x} \rrbracket$ with maximal ideal $\widehat{\mathfrak{m}}$. The $\mathbb{C}$-linear derivations of $\mathcal{O}$ form the module Der $:=\operatorname{Der}_{n}:=\operatorname{Der}_{\mathbb{C}} \mathcal{O}_{n}$ of vector fields. It is a free $\mathcal{O}$-module of rank $n$ with basis the partial derivatives $\underline{\partial}=\left(\partial_{1}, \ldots, \partial_{n}\right)$.

The module Der acts naturally on $\mathcal{O}$ and on itself by the Lie bracket $\delta(\eta):=[\delta, \eta]$ where $\delta, \eta \in$ Der. Weights $\underline{w}=\left(w_{1}, \ldots, w_{n}\right) \in \mathbb{C}^{n}$ define a vector field $\sigma=\sum_{i} w_{i} x_{i} \partial_{i} \in$ Der. A power series $p \in \mathcal{O}$ or a vector field $\delta \in$ Der is $\underline{w}$ - or $\sigma$-homogeneous of degree $\lambda \in \mathbb{C}$ if $\sigma(p)=\lambda \cdot p$ or $\sigma(\delta)=[\sigma, \delta]=\lambda \cdot \delta$. When referring to the standard weights $\underline{w}=(1, \ldots, 1)$, we omit $\underline{w}$. In this case, the $x_{i}$ are homogeneous of degree 1 , the $\partial_{i}$ of degree -1 .

Notation 2.1. Any vector field $\delta \in$ Der can be uniquely written as $\delta=\sum_{i=-1}^{\infty} \delta_{i}$ where $\delta_{i}$ is homogeneous of degree $i$ and $\delta_{0}=\underline{x} A \bar{\partial}$ for a unique matrix $A \in \mathbb{C}^{n \times n}$. For $\delta \in \mathfrak{m} \cdot$ Der, we call $\delta_{0}$ the linear part of $\delta$.

Lemma 2.2. We have $[\underline{x} A \bar{\partial}, \underline{x} B \bar{\partial}]=\underline{x}[A, B] \bar{\partial}$.

Proof. This follows immediately from $\bar{\partial}(\underline{x})=\left(\partial_{i}\left(x_{j}\right)\right)_{i, j}=\left(\delta_{i, j}\right)_{i, j}$.

Definition 2.3. Let $\delta=\sum_{i=0}^{\infty} \delta_{i} \in \mathfrak{m} \cdot$ Der and let $A \in \mathbb{C}^{n \times n}$ be such that $\delta_{0}=\underline{x} A \bar{\partial}$. Then $\delta$ is called semisimple (respectively diagonal) if $\delta=\delta_{0}$ and $A$ is semisimple (respectively diagonal). It is called nilpotent if $A$ is nilpotent.

Lemma 2.4. A nilpotent $\delta \in \mathfrak{m} \cdot$ Der is nilpotent on $\mathcal{O} / \mathfrak{m}^{k}$ and Der $/ \mathfrak{m}^{k} \cdot$ Der for all $k \geqslant 0$.

Proof. Let $\delta \in \mathfrak{m} \cdot$ Der where $\delta_{0}=\underline{x} A \bar{\partial}$ and $A \in \mathbb{C}^{n \times n}$ is nilpotent. After a $\mathbb{C}$-linear coordinate change, we may assume that $A$ has Jordan normal form. Order the monomials first by minimal degree in $\underline{x}$ and then lexicographically by $\partial_{1}>\cdots>\partial_{n}>x_{n}>\cdots>x_{1}$. Then

$$
\begin{aligned}
x_{i} \partial_{i+1}\left(x_{1}^{\alpha_{1}} \cdots x_{n}^{\alpha_{n}}\right) & =\alpha_{i+1} x_{1}^{\alpha_{1}} \cdots x_{i}^{\alpha_{i}+1} x_{i+1}^{\alpha_{i+1}-1} \cdots x_{n}^{\alpha_{n}}<x_{1}^{\alpha_{1}} \cdots x_{n}^{\alpha_{n}}, \\
x_{i} \partial_{i+1}\left(\underline{x} \underline{\underline{\alpha}} \partial_{j}\right) & =x_{i} \partial_{i+1}(\underline{x} \underline{\underline{\alpha}}) \partial_{j}-\delta_{i, j} \underline{x} \underline{\underline{\alpha}} \partial_{i+1},
\end{aligned}
$$

and $x_{i} \partial_{i+1}(\underline{x} \underline{\alpha}) \partial_{j}, \underline{x} \underline{\alpha} \partial_{j+1}<\underline{x} \underline{\alpha} \partial_{j}$, which implies the claim.

Recall that $\delta_{0}$ is the linear part of $\delta \in \mathfrak{m} \cdot$ Der.

Notation 2.5. Any vector field $\delta \in \mathfrak{m}$. Der can be uniquely written as $\delta=\delta_{S}+\delta_{N}$ where $\delta_{S}$ is semisimple, $\delta_{N}$ is nilpotent and $\left[\delta_{S, 0}, \delta_{N, 0}\right]=0$. Note that $\delta_{S}=\delta_{S, 0}$ and $\delta_{N}=\delta_{N, 0}+\sum_{i=1}^{\infty} \delta_{i}$.

Note that semisimplicity (respectively nilpotency) of $\delta \in \mathfrak{m} \cdot$ Der means that $\delta=\delta_{S}$ (respectively $\left.\delta=\delta_{N}\right)$. By Lemma 2.2, for weights $\underline{w} \in \mathbb{C}^{n}$ and a $\underline{w}$-homogeneous $\delta \in \mathfrak{m} \cdot$ Der of degree 0 , there is a $\underline{w}$-homogeneous $\mathbb{C}$-linear coordinate change such that $\delta_{0}$ is in Jordan normal form. In particular, one can always assume that $\delta_{S}$ is diagonal in this case.

Lemma 2.6. Let $\underline{w}=\left(w_{1}, \ldots, w_{n}\right) \in \mathbb{Q}^{n}$ be rational weights and let $\delta \in \mathfrak{m} \cdot$ Der be a $\underline{w}$-homogeneous vector field of degree $\lambda \in \mathbb{Q}^{*}$. Then $\delta$ is nilpotent.

Proof. Let $\sigma:=\sum_{i} w_{i} x_{i} \partial_{i}=: \underline{x} D \bar{\partial} \in$ Der where $D \in \mathbb{Q}^{n \times n}$ is diagonal and $A=\left(a_{i, j}\right)_{i, j} \in \mathbb{C}^{n \times n}$ such that $\delta_{0}=\underline{x} A \bar{\partial}$. By Lemma 2.2, $\sigma(\delta)=\lambda \cdot \delta$ implies that

$$
\left(\left(w_{i}-w_{j}\right) \cdot a_{i, j}\right)_{i, j}=[D, A]=\lambda \cdot A=\left(\lambda \cdot a_{i, j}\right)_{i, j} .
$$

We may assume that $\lambda>0$ and $w_{1} \geqslant \cdots \geqslant w_{n}$. However, then $a_{i, j}=0$ for $i \leqslant j$ and hence $\delta$ is nilpotent. 


\section{ON THE FORMAL STRUCTURE OF LOGARITHMIC VECTOR FIELDS}

Nilpotency of vector fields is clearly invariant under arbitrary coordinate changes. We shall see that diagonal vector fields are invariant under coordinate changes, which are homogeneous for the corresponding weights.

Lemma 2.7. Let $\sigma=\sum_{i} w_{i} x_{i} \partial_{i} \in$ Der and $\underline{w}:=\left(w_{1}, \ldots, w_{n}\right) \in \mathbb{C}^{n}$. Then $\sigma$ is invariant under $\underline{w}$-homogeneous coordinate changes.

Proof. Let $y_{i}=x_{i}+h_{i}$ with $\sigma\left(h_{i}\right)=w_{i} h_{i}$. Then $\partial_{x_{i}}=\partial_{y_{i}}+\sum_{j}\left(\partial h_{j} / \partial x_{i}\right) \partial_{y_{j}}$ and hence

$$
\begin{aligned}
\sum_{i} w_{i} y_{i} \partial_{y_{i}} & =\sum_{i} w_{i}\left(x_{i}+h_{i}\right)\left(\partial_{x_{i}}-\sum_{j} \frac{\partial h_{j}}{\partial x_{i}} \partial_{y_{j}}\right) \\
& =\sum_{i} w_{i} x_{i} \partial_{x_{i}}+w_{i} h_{i} \partial_{x_{i}}-\sum_{i, j} w_{i} x_{i} \frac{\partial h_{j}}{\partial x_{i}} \partial_{y_{j}}+w_{i} h_{i} \frac{\partial h_{j}}{\partial x_{i}} \partial_{y_{j}} \\
& =\sum_{i} w_{i} x_{i} \partial_{x_{i}}+w_{i} h_{i} \partial_{x_{i}}-\sum_{j} w_{j} h_{j} \partial_{y_{j}}-\sum_{i} w_{i} h_{i}\left(\partial_{x_{i}}-\partial_{y_{i}}\right) \\
& =\sum_{i} w_{i} x_{i} \partial_{x_{i}} .
\end{aligned}
$$

\section{Logarithmic vector fields}

Let $0 \neq f \in \mathcal{O}$ be a convergent power series. There are analog definitions and statements as in this section for a formal power series $0 \neq f \in \widehat{\mathcal{O}}$.

Definition 3.1. The $\mathcal{O}$-module of logarithmic vector fields is defined by

$$
\operatorname{Der}_{f}:=\operatorname{Der}_{f} \mathcal{O}:=\{\delta \in \operatorname{Der} \mid \delta(\mathcal{O} \cdot f) \subseteq \mathcal{O} \cdot f\} .
$$

If $\operatorname{Der}_{f} \not \mathbb{m}$. Der, then we call $f$ a product (with a smooth factor). If $\chi(f)=f$ for some $\chi \in \operatorname{Der}_{f}$, then $f$ is called Euler homogeneous and $\chi$ is called an Euler vector field (for $f$ ). We call $f$ strongly Euler homogeneous (at the origin) and $\chi$ a strong Euler vector field (for $f$ ) if $\chi \in \mathfrak{m} \cdot$ Der.

Under multiplication of $f$ by units, $\operatorname{Der}_{f}$ is invariant and the Lie bracket on Der induces a Lie bracket on $\operatorname{Der}_{f}$. By the Leibniz rule,

$$
\operatorname{Der}_{f}=\{\delta \in \operatorname{Der} \mid \delta(f) \in \mathcal{O} \cdot f\}
$$

can be identified with the projection of the first syzygy module of $\partial f / \partial x_{1}, \ldots, \partial f / \partial x_{n}, f$ to the first $n$ components. In particular,

$$
\operatorname{Der}_{f} \widehat{\mathcal{O}}=\widehat{\operatorname{Der}_{f} \mathcal{O}}
$$

is the $\mathfrak{m}$-adic completion of $\operatorname{Der}_{f} \mathcal{O}$ and $f$ being a product is invariant under completion. Euler homogeneity of $f$ is equivalent to $\delta(f) \notin \mathfrak{m} \cdot f$ for some $\delta \in \operatorname{Der}_{f}$. In particular, Euler homogeneity is invariant under completion and strong Euler homogeneity at the origin as well. Moreover, strong Euler homogeneity of $f$ is invariant under multiplication of $f$ by units. Indeed, if $\chi \in \operatorname{Der}_{f}$ is a strong Euler vector field for $f$ and $u \in \mathcal{O}^{*}$ then $u(u+\chi(u))^{-1} \chi$ is a strong Euler vector field for $u \cdot f$.

Lemma 3.2. If $f \in \mathcal{O}_{n}$ is a product, then there is a coordinate change such that $f=u \cdot f^{\prime}$ for some unit $u \in \mathcal{O}_{n}^{*}$ and some $f^{\prime} \in \mathcal{O}_{n-1}$. In this case,

$$
\operatorname{Der}_{f} \mathcal{O}_{n}=\mathcal{O}_{n} \cdot \operatorname{Der}_{f^{\prime}} \mathcal{O}_{n-1} \oplus \mathcal{O}_{n} \cdot \partial_{n},
$$

$\partial_{n}$ is an Euler vector field for $\exp \left(x_{n}\right) \cdot f^{\prime}$ and the strong Euler homogeneity of $f$ and $f^{\prime}$ are equivalent. 


\section{Granger And M. Schulze}

Proof. A more general version of the first statement is given by Saito [Sai80, Lemma 3.5]. If $\chi=$ $\chi^{\prime}+a_{n} \partial_{n} \in \mathfrak{m} \cdot$ Der where $\chi^{\prime} \in \mathfrak{m}_{n} \cdot \operatorname{Der}_{n-1}$ and $a_{n} \in \mathfrak{m}$ is an Euler vector field for $f^{\prime}$, then $\chi_{\mid x_{n}=0}^{\prime} \in \mathfrak{m}_{n-1} \cdot \operatorname{Der}_{n-1}$ is also an Euler vector field for $f^{\prime}$, which implies the last statement.

Lemma 3.3. Strong Euler vector fields are non-nilpotent.

Proof. Choose $k$ such that $f \in \mathfrak{m}^{k} \backslash \mathfrak{m}^{k+1}$. Then $[f] \in \mathfrak{m}^{k} / \mathfrak{m}^{k+1}$ is an eigenvector with eigenvalue 1 of any strong Euler vector field. Therefore, such a vector field cannot be nilpotent by Lemma 2.4.

Lemma 3.4. We have $\operatorname{Der}_{g \cdot h}=\operatorname{Der}_{g} \cap \operatorname{Der}_{h}$ for all $f, g \in \mathcal{O}$.

Proof. Let $f=f_{1}^{l_{1}} \cdots f_{m}^{l_{m}}$ be a decomposition of $f \in \mathcal{O}$ into irreducible factors. Then

$$
\delta(f)=\sum_{i=1}^{m} l_{i} \cdot \delta\left(f_{i}\right) \cdot f / f_{i},
$$

for all $\delta \in$ Der, and hence $\operatorname{Der}_{f}=\bigcap_{i=1}^{m} \operatorname{Der}_{f_{i}}$.

\section{Freeness and Saito's criterion}

Definition 4.1. We call a reduced $f \in \mathcal{O}$ free if $\operatorname{det}\left(\delta_{i}\left(x_{j}\right)\right)_{i, j} \in \mathcal{O}^{*} \cdot f$ for some elements $\underline{\delta}=$ $\left(\delta_{1}, \ldots, \delta_{n}\right) \in \operatorname{Der}_{f}$. Note that $\bar{\delta}=A \bar{\partial}$ for $A:=\left(\delta_{i}\left(x_{j}\right)\right)_{i, j} \in \mathcal{O}^{n \times n}$ and $\bar{\delta}:=\left(\delta_{1}, \ldots, \delta_{n}\right) \in \operatorname{Der}_{f}$. We define freeness of $f \in \widehat{\mathcal{O}}$ analogously.

Freeness of $f$ is invariant under coordinate changes, multiplication of $f$ by a unit and completion. By Saito's criterion [Sai80, Theorem 1.8.ii], a convergent $f \in \mathcal{O}$ is free if and only if $\operatorname{Der}_{f}$ is a free $\mathcal{O}$-module and $\underline{\delta}$ in Definition 4.1 is an $\mathcal{O}$-basis of $\operatorname{Der}_{f}$. One of these implications also holds for a formal $f \in \widehat{\mathcal{O}}$.

Proposition 4.2 (Formal Saito's criterion). If $f \in \widehat{\mathcal{O}}$ is free then $\operatorname{Der}_{f}$ is a free $\widehat{\mathcal{O}}$-module of rank $n$ and $\underline{\delta}$ in Definition 4.1 is an $\widehat{\mathcal{O}}$-basis of $\operatorname{Der}_{f}$.

Proof. The statement is obvious for $f \in \widehat{\mathcal{O}}^{*}$ and we may assume that $f \in \widehat{\mathfrak{m}}$. We first prove that $f \mid \operatorname{det} A$ for any $\bar{\delta} \in \operatorname{Der}_{f}^{n}$ and $A$ as in Definition 4.1. Let $\bar{a}:=\bar{\delta}(f) / f \in \widehat{\mathcal{O}}^{n}, B \in \widehat{\mathcal{O}}^{n \times n}$ the adjoint matrix of $A$, and $f=f_{1} \cdots f_{k}$ a decomposition of $f$ into different irreducible factors $f_{i} \in \widehat{\mathfrak{m}}$. Then $\operatorname{det} A \cdot \bar{\partial}(f)=B \bar{a} \cdot f$ and hence

$$
f \mid \sum_{i=1}^{k} \operatorname{det} A \cdot f_{1} \cdots \partial_{j}\left(f_{i}\right) \cdots f_{k}
$$

for all $j=1, \ldots, n$. Then $f_{i} \mid \operatorname{det} A \cdot f_{1} \cdots \partial_{j}\left(f_{i}\right) \cdots f_{k}$ for all $i=1, \ldots, k$ and $j=1, \ldots, n$. For some $j, f_{i} \mid \partial_{j}\left(f_{i}\right)$ is impossible and hence $f_{i} \mid \operatorname{det} A$ for all $i=1, \ldots, k$ and finally $f \mid \operatorname{det} A$.

Now assume that $\operatorname{det} A \in \widehat{\mathcal{O}}^{*} \cdot f$. Then $\underline{\delta}$ is $\widehat{\mathcal{O}}$-linearly independent. Let $\delta \in \operatorname{Der}_{f}$ and $\bar{b} \in \widehat{\mathcal{O}}^{n}$ such that $\delta=\underline{b} \bar{\partial}$. Then $f \mid \underline{b} B$ by the preceding arguments and hence

$$
\delta=\underline{b} \bar{\partial}=f^{-1} \underline{b} B A \bar{\partial}=f^{-1} \underline{b} B \bar{\delta} \in\langle\underline{\delta}\rangle .
$$

Thus, $\underline{\delta}$ generates $\operatorname{Der}_{f}$ and is an $\widehat{\mathcal{O}}$-basis.

\section{Formal structure theorem}

This section concerns only formal power series. The results of Saito in [Sai71, $\S \S 2-3]$ are compatible with multiweights $W=\left(\underline{w}^{1}, \ldots, \underline{w}^{s}\right)$ where $\underline{w}^{i}=\left(w_{1}^{i}, \ldots, w_{n}^{i}\right) \in \mathbb{C}^{n}$ in the following sense. 


\section{ON THE FORMAL STRUCTURE OF LOGARITHMIC VECTOR FIELDS}

Lemma 5.1 (Saito [Sai71, Lemma 2.3.iii]). Any $p \in \widehat{\mathcal{O}}$ can be uniquely written as $p=\sum_{\underline{\lambda} \in \mathbb{C}^{s}} p_{\underline{\lambda}}$ where $p_{\underline{\lambda}} \in \widehat{\mathcal{O}}$ is $W$-multihomogeneous of degree $\underline{\lambda}$.

Lemma 5.2 (Saito [Sai71, Korollar 2.5]). Let $\delta=\delta_{0} \in$ Der be linear and $W$-multihomogeneous of degree $\underline{0}$. Assume that its semisimple part $\delta_{S}=\sum_{i} w_{i} x_{i} \partial_{i}$ is diagonal and set $\underline{w}:=\left(w_{1}, \ldots, w_{n}\right)$ $\in \mathbb{C}^{n}$. Then, for any $W$-multihomogeneous $p \in \widehat{\mathcal{O}}$ of degree $\underline{\lambda} \in \mathbb{C}^{s}$ and any $\lambda \in \mathbb{C}$, there is a $W$-multihomogeneous $q \in \widehat{\mathcal{O}}$ of degree $\underline{\lambda}$ such that $\delta(q)-\lambda \cdot q+p$ is $\underline{w}$-homogeneous of degree $\lambda$.

Proof. In the proof of [Sai71, Lemma 2.4], we only need to replace the space $W_{m, \lambda}$ of homogeneous polynomials of degree $m$ that are $\underline{w}$-homogeneous of degree $\lambda$ by the subspace $W_{m, \underline{\lambda}, \lambda} \subseteq W_{m, \lambda}$ of $W$-multihomogeneous elements of degree $\underline{\lambda}$. As this space is stable by $\delta$, the same linear algebra argument applies and the claim follows exactly in the same way as in [Sai71, Korollar 2.5].

Theorem 5.3 (Saito [Sai71, Satz 3.1]). Let $\delta \in \widehat{\mathfrak{m}} \cdot$ Der be $W$-multihomogeneous of degree $\underline{0}$, $\delta_{S}=\sum_{i} w_{i} x_{i} \partial_{i}$ and $\underline{w}:=\left(w_{1}, \ldots, w_{n}\right) \in \mathbb{C}^{n}$. Then $\delta$ is $\underline{w}$-homogeneous of degree 0 after a $W$-homogeneous coordinate change. In particular, $\left[\delta_{S}, \delta_{N}\right]=0$ in this case.

Proof. The same proof as in [Sai71, Satz 3.1] works with a sequence of coordinate changes $x_{i}^{(m)}=$ $x_{i}^{(m-1)}+h_{i}$ tangent to the identity. We only need to add the condition that each $h_{i}$ is $W$-multihomogeneous of the same degree as $x_{i}$. In fact, this follows from the recursion formulas used by Saito, precisely because of Lemma 5.2, with the multihomogeneity of the coefficients in

$$
\delta=\sum g_{i}^{(m)}\left(\underline{x}^{(m)}\right) \cdot \frac{\partial}{\partial x_{i}^{(m)}}
$$

proved simultaneously.

The following result is a formal structure theorem for Der $f$. For reduced convergent $f$, Der $f$ depends only on the zero set of $f$ or the divisor defined by $f$. Considering this divisor means to consider $f$ up to contact equivalence, which allows coordinate changes and multiplication of $f$ by units. However, invariance of $\operatorname{Der}_{f}$ under contact equivalence also holds for non-reduced and formal $f$. We define the formal divisor $\widehat{\mathrm{V}}(f)$ associated with $f$ as the formal contact equivalence class of $f$. The invariant $s$ defined below can be considered as the maximal multihomogeneity of an equation of this formal divisor.

Let $0 \neq f \in \widehat{\mathcal{O}}$ be a formal power series. We assume that $f$, considered as $\widehat{\mathrm{V}}(f)$, is not a product which means, by definition, that $\operatorname{Der}_{f} \subseteq \mathfrak{m} \cdot$ Der.

Theorem 5.4 (Formal structure theorem). Let $s$ be the maximal dimension of the vector space of diagonal $\sigma \in \operatorname{Der}_{f}$ with $\sigma(f) \in \mathbb{C} \cdot f$, for $f$ varying in a formal contact equivalence class. This means that $s$ is maximal for all coordinate systems and changes of $f$ by a factor in $\widehat{\mathcal{O}}^{*}$. Then there are $\sigma_{1}, \ldots, \sigma_{s}, \nu_{1}, \ldots, \nu_{r} \in \operatorname{Der}_{f}$, a coordinate change, a change of $f$ by a factor in $\widehat{\mathcal{O}}^{*}$, and a set of irreducible factors $f_{1}, \ldots, f_{m}$ of $f$ such that:

(1) $\sigma_{1}, \ldots, \sigma_{s}, \nu_{1}, \ldots, \nu_{r}$ is a minimal system of generators of $\operatorname{Der}_{f}$;

(2) if $\delta \in \operatorname{Der}_{f}$ with $\left[\sigma_{i}, \delta\right]=0$ for all $i$, then $\delta_{S} \in\left\langle\sigma_{1}, \ldots, \sigma_{s}\right\rangle_{\mathbb{C}}$;

(3) $\sigma_{i}$ is diagonal with eigenvalues in $\mathbb{Q}$;

(4) $\nu_{i}$ is nilpotent;

(5) $\left[\sigma_{i}, \nu_{j}\right] \in \mathbb{Q} \cdot \nu_{j} ;$ and

(6) $\sigma_{i}\left(f_{j}\right) \in \mathbb{Q} \cdot f_{j}$.

Proof. Let $\sigma_{1}, \ldots, \sigma_{s} \in \operatorname{Der}_{f}$ where $\sigma_{i}=\sum_{j} w_{j}^{i} x_{j} \partial_{j}$ and $w_{j}^{i} \in \mathbb{C}$ such that $\sigma_{i}(f) \in \mathbb{C} \cdot f$. By [Sai71, Lemma 1.4], we may assume that $\underline{w}^{i}:=\left(w_{1}^{i}, \ldots, w_{n}^{i}\right) \in \mathbb{Q}^{n}$ and we denote $W:=\left(\underline{w}^{1}, \ldots, \underline{w}^{s}\right)$. Then $f$ 


\section{Granger And M. Schulze}

is $W$-multihomogeneous of some degree $\underline{\lambda}=\left(\lambda_{1}, \ldots, \lambda_{s}\right) \in \mathbb{Q}^{s}$. Let $\delta \in \operatorname{Der}_{f} \backslash\left\langle\sigma_{1}, \ldots, \sigma_{s}\right\rangle_{\mathbb{C}}$ and $a \in \widehat{\mathcal{O}}$ such that $\delta(f)=a \cdot f$. By Lemma 5.1, we may assume that $\delta$ and $a$ are $W$-multihomogeneous of some degree $\underline{\mu} \in \mathbb{Q}^{s}$. By Lemma 2.6, $\delta$ is nilpotent if $\underline{\mu} \neq 0$ and we may hence assume that $\underline{\mu}=\underline{0}$. By Theorem $5 . \overline{3}$, there is a $W$-multihomogeneous coordinate change such that $\delta$ is $\underline{w}$-homogeneous of degree 0 where $\delta_{S}=\sum_{i} w_{i} x_{i} \partial_{i}$ and $\underline{w}:=\left(w_{1}, \ldots, w_{n}\right) \in \mathbb{C}^{n}$. By Lemma 2.7, the $\sigma_{i}$ are invariant under this coordinate change.

We shall multiply $f$ by a $W$-multihomogeneous $u \in \widehat{\mathcal{O}}^{*}$ of degree $\underline{0}$ to make $a \underline{w}$-homogeneous of degree 0 . The transformation of $a$ under this operation is given by

$$
\delta(u f)=\left(\delta(u) \cdot u^{-1}+a\right) \cdot u f .
$$

Let $d \geqslant 1$ be the minimal degree in which $a$ is not $\underline{w}$-homogeneous of degree 0 . If $u=1+u_{d}$ where $u_{d}$ is homogeneous of degree $d$ then $\delta_{0}\left(u_{d}\right)+a_{d}$ is the degree $d$ part of the transformed $a$. By Lemma 5.2, there is a $W$-multihomogeneous $u_{d}$ of degree $\underline{0}$ such that $\delta_{0}\left(u_{d}\right)+a_{d}$ is $\underline{w}$-homogeneous of degree 0 . Then the desired $u$ exists by induction on $d$. We may hence assume that $a$ is $\underline{w}$-homogeneous of degree 0 .

Let $f=\sum_{\lambda} f_{\lambda}$ be the expansion of $f$ in $\underline{w}$-homogeneous parts as in Lemma 5.1. Then $\delta\left(f_{\lambda}\right)=$ $a \cdot f_{\lambda}$ and hence $\delta_{N}\left(f_{\lambda}\right)=(a-\lambda) \cdot f_{\lambda}$ for all $\lambda$. By Lemma 2.4, $f_{\lambda}=0$ for $\lambda \neq a_{0}$ and hence $f=f_{a_{0}}$. However, then $\delta_{S}(f)=a_{0} \cdot f$ and hence, by the minimality assumption, $\delta_{S} \in\left\langle\sigma_{1}, \ldots, \sigma_{s}\right\rangle_{\mathbb{C}}$. So we can assume that $\delta=\delta_{N}$ is nilpotent.

It remains to construct the $W$-homogeneous irreducible factors of $f$. Let $W^{\prime}:=\left(\underline{w}^{1}, \ldots, \underline{w}^{s^{\prime}}\right)$ where $s^{\prime} \leqslant s$ is maximal such that there is a set of irreducible $W^{\prime}$-homogeneous factors $f_{1}, \ldots, f_{m}$ of $f$. We may assume that $m \geqslant 2$ and $s^{\prime}<s$. Let $t:=s^{\prime}+1, \sigma_{t}=\sum_{i} w_{i} x_{i} \partial_{i}, \underline{w}:=\left(w_{1}, \ldots, w_{n}\right) \in \mathbb{Q}^{n}$, and $f=f_{1}^{l_{1}} \cdots f_{m}^{l_{m}}$. By Lemma 3.4, $\sigma_{t}\left(f_{i}\right)=a_{t, i} \cdot f_{i}$ for some $a_{t, i} \in \widehat{\mathcal{O}}$. By the above argument, there are, for $i=1, \ldots, m-1, W^{\prime}$-multihomogeneous $u_{t, i} \in \widehat{\mathcal{O}}^{*}$ and $\lambda_{t, i} \in \mathbb{Q}$ such that

$$
\sigma_{t}\left(u_{t, i} f_{i}\right)=\lambda_{t, i} \cdot u_{t, i} f_{i}
$$

We choose $u_{t, m} \in \widehat{\mathcal{O}}^{*}$ such that $\prod_{i=1}^{m} u_{t, i}^{l_{i}}=1$ and set $\lambda_{t, m}:=l_{m}^{-1} \cdot\left(\lambda_{t}-\sum_{i=1}^{m-1} l_{i} \lambda_{t, i}\right) \in \mathbb{Q}$. Then (1) holds for $i=1, \ldots, m$ and hence $u_{t, 1} f_{1}, \ldots, u_{t, m} f_{m}$ form a set of $W^{\prime}$-multihomogeneous, $\underline{w}$-homogeneous, irreducible factors of $f$. This contradicts the maximality of $s^{\prime}$ and finishes the proof.

Corollary 5.5. If $f$ is Euler homogeneous then at least one $\sigma_{i}$ in Theorem 5.4 can be chosen to be an Euler vector field.

Proof. This follows immediately from Lemma 2.4.

Corollary 5.6. If $\operatorname{Der}_{f}$ is a free $\widehat{\mathcal{O}}$-module then there is a basis of $\operatorname{Der}_{f}$ as in Theorem 5.4.

Proof. This follows immediately from Nakayama's lemma.

Corollary 5.7. In Theorem 5.4, if $f$ is free and $\lambda_{j}^{i} \in \mathbb{Q}$ such that $\left[\sigma_{i}, \nu_{j}\right]=\lambda_{j}^{i} \cdot \nu_{j}$, then $f$ is $\sigma_{i}$-homogeneous of degree $\sum_{j=1}^{n} w_{j}^{i}+\sum_{j=1}^{n-s} \lambda_{j}^{i}$.

Proof. This follows immediately from Definition 4.1 .

\section{Formal Lie algebra structure}

This section concerns only formal power series. Let $0 \neq f \in \widehat{\mathcal{O}}$ be not a product and let $\sigma_{1}, \ldots, \sigma_{s}$, $\nu_{1}, \ldots, \nu_{r} \in \operatorname{Der}_{f}$ be as in Theorem 5.4. Let $\mathfrak{D}_{d}$ be the Lie algebra $\operatorname{Der}_{f} / \mathfrak{m}^{d} \cdot \operatorname{Der}_{f}$ over $\mathbb{C}$ where $d \geqslant 1$. 


\section{ON THE FORMAL STRUCTURE OF LOGARITHMIC VECTOR FIELDS}

In this section, we freely denote by the same letter a vector field $\delta \in \operatorname{Der}_{f}$ and its class modulo $\mathfrak{m}^{d} \cdot \operatorname{Der}_{f}, \delta \in \mathfrak{D}_{d}$. Then $\mathfrak{S}_{d}:=\bigoplus_{i=1}^{s} \mathbb{C} \cdot \sigma_{i} \subseteq \mathfrak{D}_{d}$ is an abelian Lie subalgebra. The centralizer $\mathfrak{C}\left(\mathfrak{S}_{d}\right)$ of $\mathfrak{S}_{d}$ in $\mathfrak{D}_{d}$ is the Lie subalgebra of $\underline{\sigma}$-multihomogeneous logarithmic vector fields of degree $\underline{0}$ where $\underline{\sigma}:=\left(\sigma_{1}, \ldots, \sigma_{s}\right)$. By Theorem $5.4, \overline{\mathfrak{C}}\left(\mathfrak{S}_{d}\right)$ is an almost algebraic Lie algebra [Jac62, III.11]. The derived series of $\mathfrak{D}_{d}$ is defined by

$$
\mathfrak{D}_{d}^{(0)}:=\mathfrak{D}_{d}, \quad \mathfrak{D}_{d}^{(i+1)}=\left[\mathfrak{D}_{d}^{(i)}, \mathfrak{D}_{d}^{(i)}\right]
$$

and $\mathfrak{D}_{d}$ is called solvable if $\mathfrak{D}_{d}^{(i)}=0$ for $i \gg 0$ (see [Jac62, I.7]). By Lemma 2.4 and Engel's theorem $\left[\right.$ Jac62, II.3], $\mathfrak{m} \cdot \mathfrak{D}_{d}$ is a nilpotent ideal and hence $\mathfrak{D}_{d}$ is solvable if and only if $\mathfrak{D}_{d} / \mathfrak{m} \cdot \mathfrak{D}_{d}=\mathfrak{D}_{1}$ is solvable. An element $\delta \in \mathfrak{D}_{1}$ is reduced to its linear part $\delta=\underline{x} A \bar{\delta}$ where $A \in \mathbb{C}^{n \times n}$. In the rest of this section, we hence assimilate $\delta$ to the matrix $A$.

Proposition 6.1. The Lie algebras $\mathfrak{D}_{d}$ are solvable if $r \leqslant 1$ or $s=0$ in Theorem 5.4. In particular, this holds if $\operatorname{Der}_{f}$ is a free $\widehat{\mathcal{O}}$-module of rank 2 .

Proof. If $r=0$, then $\mathfrak{D}_{1}=\mathfrak{S}_{1}$ is abelian. If $r=1$, then $\mathfrak{D}_{1}=\mathfrak{S}_{1} \oplus \mathbb{C} \cdot \nu_{1}$ and $\mathbb{C} \cdot \nu_{1} \subseteq \mathfrak{D}_{1}$ is a nilpotent ideal. If $s=0$, then $\mathfrak{D}_{1}$ is nilpotent by Theorem 5.4.(2) and Engel's theorem [Jac62, II.3]. The second claim follows from Corollary 5.6.

In the following, we prove the solvability of $\mathfrak{D}_{1}$ for a free $f$ in dimension $n=3$. By Proposition 6.1, it suffices to consider the case $s=1$ in Theorem 5.4. In a convenient system of coordinates $(x, y, z)$, $\operatorname{Der}_{f}$ is generated by a diagonal vector field $\sigma=a x \partial_{x}+b y \partial_{y}+c z \partial_{z}$ where $a, b, c \in \mathbb{Q}$ and two $\sigma$-homogeneous nilpotent vector fields $\nu_{1}, \nu_{2}$ of degrees $\lambda_{1}, \lambda_{2}$. The set of eigenvalues of $[\sigma, \cdot]$ is the set of differences of $a, b, c$ and includes $\lambda_{1}$ and $\lambda_{2}$. There is a $\sigma$-homogeneous relation

$$
\mu:=\left[\nu_{1}, \nu_{2}\right]=\lambda \sigma+p_{1} \nu_{1}+p_{2} \nu_{2} \quad \text { where } \lambda, p_{1}, p_{2} \in \mathbb{C}
$$

of degree $\lambda_{1}+\lambda_{2}$. As the trace of a commutator, $\operatorname{tr} \mu=0$ and, by additivity, $\operatorname{tr}(\lambda \sigma)=0$.

We first show that $\lambda \neq 0$ if the Lie algebra $\mathfrak{D}_{1}$ is not solvable. Indeed if $\lambda=0$, then $\mu=p_{1} \nu_{1}+p_{2} \nu_{2}$ and $\mathfrak{D}_{1}^{(1)} \subseteq\left\langle\nu_{1}, \nu_{2}\right\rangle$. Therefore, $\mathfrak{D}_{1}^{(2)} \subseteq \mathbb{C} \cdot \mu$ and hence $\mathfrak{D}_{1}^{(3)}=0$, which proves that $\mathfrak{D}_{1}$ is solvable. (In fact we might prove with some more calculations that already $\mu=0$.)

We may assume now that $\lambda \neq 0$. Then the $\sigma$-degree of $\lambda \sigma$ and hence of $p_{1} \nu_{1}, p_{2} \nu_{2}$, and $\mu$ equals 0 . In particular, $\lambda_{i} \neq 0$ implies that $p_{i}=0$ for $i=1,2$ and $\lambda_{1}+\lambda_{2}=0$ being the $\sigma$-degree of $\mu$. Finally, the situation of a non-solvable $\mathfrak{D}_{1}$ reduces to the following two cases:

- Case I: $\mu=\lambda \sigma+p_{1} \nu_{1}+p_{2} \nu_{2}$ where $\lambda \neq 0$ and $\lambda_{1}=\lambda_{2}=0$;

- Case II: $\mu=\lambda \sigma$ where $\lambda \neq 0$ and $\lambda_{1}=-\lambda_{2} \neq 0$.

In Case I, we would have $\mathfrak{D}_{1}^{(1)}=\mathbb{C} \cdot \mu$ and $\mathfrak{D}_{1}$ would be solvable as follows from $\mathfrak{D}_{1}^{(2)}=0$. However, we prove easily that Case I cannot occur. We also prove that Case II is impossible by a more complicated argument.

Case I: $\mu=\lambda \sigma+p_{1} \nu_{1}+p_{2} \nu_{2}$ where $\lambda \neq 0$ and $\lambda_{1}=\lambda_{2}=0$.

We may assume that $a \neq 0$ and let $E$ be the $a$-eigenspace of $\sigma$. The equality $\lambda_{i}=0$ means that the $\nu_{i}$ commute with $\sigma$ and that $E$ is invariant under the $\nu_{i}$ and hence under $\mu$. By restricting to $E$, we obtain the contradiction $0=\operatorname{tr}\left(\sigma_{\mid E}\right)=a \cdot \operatorname{dim} E$.

Case II: $\mu=\lambda \sigma$ where $\lambda \neq 0$ and $\lambda_{1}=-\lambda_{2} \neq 0$.

Subcase (a): $\sigma=a\left(x \partial_{x}+y \partial_{y}+z \partial_{z}\right)$. This is impossible since all $\sigma$-homogeneous vector fields are of degree $a-a=0$. 


\section{Granger And M. Schulze}

Subcase (b): $\sigma=a x \partial_{x}+b\left(y \partial_{y}+z \partial_{z}\right)$ where $a \neq b$. We may assume that $\lambda_{1}=a-b$ and write

$$
\nu_{1}=\left(\begin{array}{ccc}
0 & r & s \\
0 & 0 & 0 \\
0 & 0 & 0
\end{array}\right) \quad \text { and } \quad \nu_{2}=\left(\begin{array}{ccc}
0 & 0 & 0 \\
u & 0 & 0 \\
v & 0 & 0
\end{array}\right) \text {. }
$$

Calculating the commutator gives

$$
\left(\begin{array}{ccc}
r u+s v & 0 & 0 \\
0 & -u r & -u s \\
0 & -v r & -v s
\end{array}\right)=\left[\nu_{1}, \nu_{2}\right]=\lambda \sigma=\lambda \cdot\left(\begin{array}{ccc}
a & 0 & 0 \\
0 & b & 0 \\
0 & 0 & b
\end{array}\right)
$$

which is equivalent to

$$
v r=u s=0 \quad \text { and } \quad(r u+s v, u r, v s)=(\lambda a, \lambda b, \lambda b) .
$$

The equations $v r=0=u s$ imply at least one of the equalities $v=0, r=0, u=0, s=0$. Each of these taken into the other relations $\lambda b=u r=v s=\frac{1}{2} \lambda a$ gives $b=0$ and then $a=0$, a contradiction.

Subcase (c): $\sigma=a x \partial_{x}+b y \partial_{y}+c z \partial_{z}$ where $a \neq b \neq c \neq a$. In this case, the relations between $\sigma, \nu_{1}, \nu_{2}$ alone do not contradict to a non-solvable $\mathfrak{D}_{1}$. We shall exclude this case by using the equation $f$.

Up to permutation, there are two cases: $\lambda_{1}=a-c$ and $\lambda_{2}=c-a$ where $\pm(c-b) \neq \lambda_{1} \neq \pm(a-b)$ or $\lambda_{1}=a-b$ and $\lambda_{2}=c-b$.

In the first case, we may assume by changing $\nu_{1}, \nu_{2}$, and $\sigma$ (or equivalently $\lambda$ ) by a constant factor that

$$
\nu_{1}=\left(\begin{array}{lll}
0 & 0 & 1 \\
0 & 0 & 0 \\
0 & 0 & 0
\end{array}\right), \quad \nu_{2}=\left(\begin{array}{lll}
0 & 0 & 0 \\
0 & 0 & 0 \\
1 & 0 & 0
\end{array}\right), \quad \sigma=\left(\begin{array}{rrr}
1 & 0 & 0 \\
0 & 0 & 0 \\
0 & 0 & -1
\end{array}\right)=\frac{1}{2}\left[\nu_{1}, \nu_{2}\right]
$$

So the linear parts of the generators of $\operatorname{Der}_{f}$ are in the canonical form

$$
\sigma=x \partial_{x}-z \partial_{z}, \quad \nu_{1}=x \partial_{z}, \quad \nu_{2}=z \partial_{x} .
$$

In the second case, we may assume that $(a, b, c)=(1,0,-1)$ after changing $\sigma$ by a constant factor as $\operatorname{tr} \sigma=0$. Then we may write

$$
\nu_{1}=\left(\begin{array}{ccc}
0 & 1 & 0 \\
0 & 0 & r \\
0 & 0 & 0
\end{array}\right) \quad \text { and } \quad \nu_{2}=\left(\begin{array}{ccc}
0 & 0 & 0 \\
s & 0 & 0 \\
0 & t & 0
\end{array}\right)
$$

Calculating the commutator gives

$$
\left(\begin{array}{ccc}
s & 0 & 0 \\
0 & r t-s & 0 \\
0 & 0 & -r t
\end{array}\right)=\left[\nu_{1}, \nu_{2}\right]=\lambda \sigma=\lambda \cdot\left(\begin{array}{ccc}
1 & 0 & 0 \\
0 & 0 & 0 \\
0 & 0 & -1
\end{array}\right)
$$

and hence $s=\lambda=r t$. By the change of coordinates $(x, y, r z)$, we reduce to the case $r=1$ and, by dividing $\nu_{2}$ by $\lambda$, to the case $s=t=1$. So the linear parts of the generators of $\operatorname{Der}_{f}$ are in the canonical form

$$
\sigma=x \partial_{x}-z \partial_{z}, \quad \nu_{1}=x \partial_{y}+y \partial_{z}, \quad \nu_{2}=y \partial_{x}+z \partial_{y} .
$$

By Corollary 5.7, we may assume that $f$ is $\sigma$-homogeneous of degree 0 with the same $\sigma$ for (2) and (3). We can hence write $f=\sum_{i \geqslant k} f_{i}$ where $f_{i}$ is homogeneous of degree $i$ and $0 \neq f_{k}=$ $\sum_{i} c_{i} x^{i} y^{k-2 i} z^{i}$. By Lemma 2.4, $\nu_{1}\left(f_{k}\right)=\nu_{2}\left(f_{k}\right)=0$. For (2), we obtain $\partial f_{k} / \partial x=\partial f_{k} / \partial z=0$ and hence $f_{k}=f_{k}(y)$ depends only on $y$. For (3), the coefficients of the equation $\nu_{1}\left(f_{k}\right)=0$ are $(k-2 i+2) c_{i-1}+i c_{i}=0$ and hence $c_{0} \neq 0$. Thus both (2) and (3) contradict $f \in\langle x, z\rangle$ by Definition 4.1. 


\section{ON THE FORMAL STRUCTURE OF LOGARITHMIC VECTOR FIELDS}

Proposition 6.2. The Lie algebras $\mathfrak{D}_{d}$ are solvable for free $f$ in dimension $n \leqslant 3$.

Proof. This follows from Proposition 6.1 and the preceding arguments.

Example 6.3. Consider the representation of the non-solvable Lie algebra $\mathbb{C} \times \mathfrak{s l}_{2}$ defined by

$$
\begin{aligned}
X & =\left(\begin{array}{llll}
1 & 0 & 0 & 0 \\
0 & 1 & 0 & 0 \\
0 & 0 & 1 & 0 \\
0 & 0 & 0 & 1
\end{array}\right), \quad H=\left(\begin{array}{rrrr}
3 & 0 & 0 & 0 \\
0 & 1 & 0 & 0 \\
0 & 0 & -1 & 0 \\
0 & 0 & 0 & -3
\end{array}\right), \\
S_{+} & =\left(\begin{array}{llll}
0 & 0 & 0 & 0 \\
1 & 0 & 0 & 0 \\
0 & 1 & 0 & 0 \\
0 & 0 & 1 & 0
\end{array}\right), \quad S_{-}=\left(\begin{array}{rrrr}
0 & -3 & 0 & 0 \\
0 & 0 & -4 & 0 \\
0 & 0 & 0 & -3 \\
0 & 0 & 0 & 0
\end{array}\right) .
\end{aligned}
$$

Let $\chi=\underline{x} X \bar{\partial}, \eta=\underline{x} H \bar{\partial}, \sigma_{ \pm}=\underline{x} S_{ \pm} \bar{\partial}$ be the corresponding vector fields. Then

$$
\left(\begin{array}{c}
\chi \\
\eta \\
\sigma_{+} \\
\sigma_{-}
\end{array}\right)=A \cdot \bar{\partial}, \quad A=\left(\begin{array}{cccc}
x_{1} & x_{2} & x_{3} & x_{4} \\
3 x_{1} & x_{2} & -x_{3} & -3 x_{4} \\
x_{2} & x_{3} & x_{4} & 0 \\
0 & -3 x_{1} & -4 x_{2} & -3 x_{3}
\end{array}\right)
$$

and $f=\frac{1}{2} \operatorname{det}(A)=3 x_{2}^{2} x_{3}^{2}-6 x_{1} x_{3}^{3}-8 x_{2}^{3} x_{4}+18 x_{1} x_{2} x_{3} x_{4}-9 x_{1}^{2} x_{4}^{2}$ is irreducible. By [Sai80, Lemma 1.9], this implies that $f$ is free and $\chi, \eta, \sigma_{+}, \sigma_{-}$is a basis of $\operatorname{Der}_{f}$. By construction, $\mathfrak{D}_{1}=\mathbb{C} \times \mathfrak{s} l_{2}$ is not solvable. So the statement of Proposition 6.2 fails in dimension $n=4$.

\section{LCT and Euler homogeneity}

Finally we return to the situation of the introduction. Let $x \in D$ and choose a coordinate system $\underline{x}=\left(x_{1}, \ldots, x_{n}\right)$ at $x$ defined in some Stein open neighborhood $V_{x}$ of $x$. Let $\mathcal{V}_{x}=\left(V_{x, i}\right)_{i=1, \ldots, n}$ be the Stein open covering of $V_{x}^{*}:=V_{x} \backslash\{x\}$ defined by $V_{x, i}:=\left\{x \in V_{x} \mid x_{i} \neq 0\right\}$ and $\mathcal{U}_{x}$ its restriction to $V_{x} \backslash D$. The following explicit necessary condition for LCT for free divisors is due to Castro-Jiménez et al. [CNM96, CMNC02]. Its proof relies on the comparison of the four spectral sequences arising from the two double complexes $\check{\mathrm{C}}^{q}\left(\mathcal{V}_{x}, \Omega_{V_{x}^{*}}^{p}(\log D)\right)$ and $\check{\mathrm{C}}^{q}\left(\mathcal{U}_{x}, \Omega_{V_{x} \backslash D}^{p}\right)$.

Theorem 7.1 (Calderón-Moreno et al. [CMNC02]). Let $D \subseteq X$ be a free divisor and assume that $L C T$ holds for $D$ in $V_{x}^{*}$ where $x \in X$. Then LCT holds for $D$ at $x$ if and only if

$$
0 \longrightarrow \check{\mathrm{H}}^{n-1}\left(V_{x}^{*}, \mathcal{O}_{X}\right) \stackrel{\mathrm{d}_{1}}{\longrightarrow} \check{\mathrm{H}}^{n-1}\left(V_{x}^{*}, \Omega_{X}^{1}(\log D)\right) \stackrel{\mathrm{d}_{2}}{\longrightarrow} \check{\mathrm{H}}^{n-1}\left(V_{x}^{*}, \Omega_{X}^{2}(\log D)\right)
$$

is an exact sequence.

We shall combine Theorems 5.4 and 7.1 to prove Conjecture 1.1 in dimension $n=3$, which is our main result Theorem 1.6. We first give a more explicit description of the map $\mathrm{d}_{1}$ in Theorem 7.1.

As $\mathcal{V}_{x}$ is a Stein open covering of $V_{x}^{*}$, one can identify

$$
\begin{aligned}
H & :=\check{\mathrm{H}}^{n-1}\left(V_{x}^{*}, \mathcal{O}_{X}\right)=\check{\mathrm{H}}^{n-1}\left(\mathcal{V}_{x}, \mathcal{O}_{X}\right) \\
& =\mathbb{C}\left\{x_{1}, x_{1}^{-1}, \ldots, x_{n}, x_{n}^{-1}\right\} / \sum_{i=1}^{n} \mathbb{C}\left\{x_{1}, x_{1}^{-1}, \ldots, x_{i}, \widehat{x_{i}^{-1}}, \ldots, x_{n}, x_{n}^{-1}\right\}
\end{aligned}
$$

where $\mathbb{C}\left\{x_{1}, x_{1}^{-1}, \ldots, x_{n}, x_{n}^{-1}\right\}$ denotes the ring of Laurent series in $x_{1}, \ldots, x_{n}$. Since the Stein open neighborhoods of $x$ form a fundamental system of neighborhoods of $x$, we may restrict our considerations to germs $X=(X, x)=\left(\mathbb{C}^{n}, 0\right), D=(D, x)=\mathrm{V}(f) \subseteq X$ where $f \in \mathfrak{m}_{X}$, and

$$
\operatorname{Der}_{X}(-\log D)=\operatorname{Der}_{f}
$$




\section{Granger And M. Schulze}

as in Definition 3.1. In the following, we abbreviate $\mathfrak{m}:=\mathfrak{m}_{X} \subseteq \mathcal{O}_{X}=: \mathcal{O}$, Der $:=$ Der $_{X}$ and $\Omega^{\bullet}:=\Omega_{X}^{\bullet}$.

Let $\underline{\omega}=\left(\omega_{1}, \ldots, \omega_{n}\right)$ be a basis of $\Omega^{1}(\log D)$ and $\underline{\delta}=\left(\delta_{1}, \ldots, \delta_{n}\right)$ its dual basis of $\operatorname{Der}(-\log D)$. Via $\underline{\omega}: \mathcal{O}^{n} \cong \Omega^{1}(\log D)$, one can identify

$$
\mathrm{d}_{1}=\left(\delta_{1}, \ldots, \delta_{n}\right): H \longrightarrow H^{n}, \quad[g] \longmapsto\left(\left[\delta_{1}(g)\right], \ldots,\left[\delta_{n}(g)\right]\right) .
$$

We only make use of the following consequence of Theorem 7.1.

Corollary 7.2. If $L C T$ holds for a free divisor $D \subseteq X$, then $\operatorname{ker} \mathrm{d}_{1}=0$.

In the following, we abbreviate

$$
X^{\prime}:=\left(\mathbb{C}^{n-1}, 0\right), \quad X^{\prime \prime}:=(\mathbb{C}, 0), \quad X:=X^{\prime} \times X^{\prime \prime}
$$

and reduce the problem to the case where $D$ is not a product with a smooth factor. We first note that freeness and strong Euler homogeneity are independent of smooth factors.

Lemma 7.3. Let $D \subseteq X$ be a divisor. Then $D \cong D^{\prime} \times X^{\prime \prime}$ for some divisor $D^{\prime} \subseteq X^{\prime}$ is equivalent to $\operatorname{Der}(-\log D) \nsubseteq \mathfrak{m} \cdot$ Der. In this case, $D$ is Euler homogeneous and each of the following properties is equivalent for $D$ and $D^{\prime}$ : strong Euler homogeneity, freeness and Koszul freeness.

Proof. This follows immediately from Lemma 3.2.

By Castro-Jiménez et al. [CNM96, Lemma 2.2.i,ii], LCT is also independent of smooth factors.

Lemma 7.4 (Castro-Jiménez et al. [CNM96]). Let $D^{\prime} \subseteq X^{\prime}$ be a divisor and $D=D^{\prime} \times X^{\prime \prime}$. Then $L C T$ for $D^{\prime}$ is equivalent to $L C T$ for $D$.

By Theorem 1.2 and Lemmas 7.3 and 7.4, we may assume from now on that $\mathrm{I}(D)=\langle f\rangle$ where $f$ is not a product as in Definition 3.1. Then it suffices to prove that $f$ is Euler homogeneous if LCT holds for $D$ and the results in the preceding sections can be applied.

We use Corollary 7.2 only in the following special case.

Lemma 7.5. Let $\delta \in \mathfrak{m} \cdot$ Der and $A \in \mathbb{C}^{n \times n}$ such that $\delta_{0}=\underline{x} A \bar{\partial}$. Then

$$
\delta\left[\frac{1}{x_{1} \cdots x_{n}}\right]=\left[\frac{\operatorname{tr} A}{x_{1} \cdots x_{n}}\right] \in H .
$$

Proof. This follows immediately from the definition of $H$.

From now on, let $n=3$ and abbreviate

$$
x, y, z:=x_{1}, x_{2}, x_{3} \quad \text { and } \quad \partial_{x}, \partial_{y}, \partial_{z}:=\partial_{x_{1}}, \partial_{x_{3}}, \partial_{x_{3}} .
$$

We assume that $f$ is not Euler homogeneous and claim that LCT does not hold for $D$. By Corollary 5.6, there is an $\widehat{\mathcal{O}}$-basis $\sigma_{1}, \ldots, \sigma_{s}, \nu_{1}, \ldots, \nu_{n-s}$ of $\operatorname{Der}_{f} \widehat{\mathcal{O}}$ as in Theorem 5.4 and, by Corollary 5.5, we may assume that $\sigma_{i}(f)=0$ for all $i=1, \ldots, s$. There are the following cases.

Case I: $s=0$.

Then the claim follows from Corollary 7.2 and Lemma 7.5 using a truncated coordinate change in Theorem 5.4. 


\section{ON THE FORMAL STRUCTURE OF LOGARITHMIC VECTOR FIELDS}

Case II: $s=1$ and $\sigma=\sigma_{1}=a x \partial_{x}+b y \partial_{y}+c z \partial_{z}$.

Subcase (a): $a \neq 0$ and $b, c=0$. In this situation, $f$ is annihilated by $\sigma=a x \partial_{x}$ and hence $\partial_{x} \in \operatorname{Der}_{f}$ in contradiction to our assumption that $f$ is not a product.

Subcase (b): $a, b \neq 0$ and $c=0$. Then $\sigma(f)=0$ implies that $a b<0$ and $f=\sum_{i a+j b=0} a_{i, j}(z) x^{i} y^{j}$. As $f \in\langle x, y\rangle$ by Definition $4.1, a_{0,0}(z)=0$. However, $f$ being reduced implies that $a_{1,1}(z) \neq 0$, which forces $a=-b$. Then the claim follows from Corollary 7.2 and Lemma 7.5 using a truncated coordinate change in Theorem 5.4.

Subcase (c): $a, b, c \neq 0$. A truncated coordinate change in Theorem 5.4 yields the existence of a (convergent) $\delta \in \operatorname{Der}_{f} \mathcal{O}$ such that $\delta_{0}=\sigma$. Then $\delta$ vanishes only at the origin and is tangent to the one-dimensional smooth part of Sing $D$. This implies that the logarithmic characteristic subvariety $\mathrm{L}_{X}(-\log D) \subseteq \mathrm{T}_{X}^{*}$ (see [Sai80, Definition 3.15]) has minimal dimension $n=3$. (In the language of Saito, the existence of $\delta$ above implies that the logarithmic stratification of $X$ consists only of holonomic strata [Sai80, Definitions 3.3 and 3.8] and hence $\mathrm{L}_{X}(-\log D)$ has only holonomic components [Sai80, Definition 3.17 and Proposition 3.18].) However, then $D$ is Koszul free by [CN02, Corollary 1.9] and LCT does not hold for $D$ by Corollary 1.5.

Case III: $s \geqslant 2$.

In this situation, there are two linearly independent $\sigma_{1}, \sigma_{2} \in \operatorname{Der}_{f} \widehat{\mathcal{O}}$ and hence the Newton diagram of $f$ is contained in a one-dimensional vector space. Then there is a monomial $x^{i} y^{j} z^{k}$ such that $f=u \cdot x^{i} y^{j} z^{k}$ for some $u \in \widehat{\mathcal{O}}^{*}$. However, this means that $D$ is Euler homogeneous in contradiction to our assumption.

Finally we have proved our main result Theorem 1.6. There is also a simple proof of Theorem 1.2 using Theorem 5.4, Corollary 7.2 and Lemma 7.5 as above.

\section{ACKnowledgements}

The second author is grateful to David Mond for helpful hints. We like to thank Tristan Torrelli for remarks on the introduction.

\section{REFERENCES}

AA88 D. V. Anosov and I. V. Arnol'd (eds), Dynamical systems I, Encyclopedia of Mathematical Sciences, vol. 1 (Springer, Berlin, 1988).

CMNC02 F. J. Calderón-Moreno, D. Mond, L. Narváez-Macarro and F. J. Castro-Jiménez, Logarithmic cohomology of the complement of a plane curve, Comment. Math. Helv. 77 (2002), 24-38.

CN02 F. J. Calderón-Moreno and L. Narváez-Macarro, The module $\mathcal{D} f^{s}$ for locally quasi-homogeneous free divisors, Comput. Math. 134 (2002), 59-74.

CN05 F. J. Calderón-Moreno and L. Narváez-Macarro, Dualité et comparaison sur les complexes de de Rham logarithmiques par rapport aux diviseurs libres, Ann. Inst. Fourier (Grenoble) $\mathbf{5 5}$ (2005), $47-75$.

CNM96 F. J. Castro-Jiménez, L. Narváez-Macarro and D. Mond, Cohomology of the complement of a free divisor, Trans. Amer. Math. Soc. 348 (1996), 3037-3049.

CU05 F. J. Castro-Jiménez and J. M. Ucha-Enríquez, Logarithmic Comparison Theorem and some Euler homogeneous free divisors, Proc. Amer. Math. Soc. 133 (2005), 1417-1422.

Del71 P. Deligne, Théorie de Hodge, II, Publ. Math. Inst. Hautes Études Sci. 40 (1971), 5-57.

Gro66 A. Grothendieck, On the de Rham cohomology of algebraic varieties, Publ. Math. Inst. Hautes Études Sci. 29 (1966), 95-103. 


\section{ON THE FORMAL STRUCTURE OF LOGARITHMIC VECTOR FIELDS}

Jac62 N. Jacobson, Lie algebras (Interscience, New York, 1962).

Sai71 K. Saito, Quasihomogene isolierte Singularitäten von Hyperflächen, Invent. Math. 14 (1971), 123-142.

Sai80 K. Saito, Theory of logarithmic differential forms and logarithmic vector fields, J. Fac. Sci. Univ. Tokyo 27 (1980), 265-291.

Tor04 T. Torrelli, On meromorphic functions defined by a differential system of order 1, Bull. Soc. Math. France 132 (2004), 591-612.

Michel Granger michel.granger@univ-angers.fr

Université d'Angers, 2 Bd. Lavoisier, 49045 Angers, France

Mathias Schulze mathias.schulze@univ-angers.fr

Université d'Angers, 2 Bd. Lavoisier, 49045 Angers, France 